\title{
16
}

\section{SOME REMARKS ON THE METHODS FORMULATED IN A RECENT ARTICLE ON “THE QUANTITATIVE ANALYSIS OF PLANT GROWTH."}

\author{
By R. A. FISHER, \\ Chief Statistician, Rothamsted Experimental Station.
}

IN the Annals of Applied Biology has appeared a paper(4) by Briggs, Kidd and West; according to the authors "The series of articles of which this is the first instalment, constitutes an attempt to formulate methods for the quantitative analysis of plant growth and to apply these methods to data which have been lying dormant in the literature for forty years."

That the paper here criticised is primarily concerned with the methods by which primary observations are to be treated in the study of plant growth is emphasised by a reference made to it in an earlier paper(3) in the New Phylologist. "The Relative Growth Rate, $R$, is the weekly percentage rate at which the dry weight increases. It may be assumed for purposes of calculation that the increase from week to week takes place exponentially, $R$ being the exponent, or that it takes place linearly. Both are approximations. As to the relative merits of the two different methods the reader is referred to (4)."

It will be noticed that no definition is here given of the Relative Growth Rate, $R$, except for the case in which the mass increases exponentially, when this is so

$$
m=m_{0} e^{R t}
$$

where $m$ is the mass and $t$ the time, and $R$ may be calculated from any two observations:

$$
R=\frac{\log m_{2}-\log m_{1}}{t_{2}-t_{1}}
$$

where the suffixes 1 and 2 indicate the first and second observations.

This is the quantity termed the Efficiency Index by Blackman(1), and which may be correctly termed the Relative Growth Rate; we shall use the latter term. No indication is given above as to what meaning is to be attached to the term Relative Growth Rate when it is assumed that the increase takes place linearly or as to how it is to be calculated from the observations. 
It is here fitting to remark that whatever assumptions are made as to the variations of growth rate with time, the relative growth rate at any instant is necessarily

$$
\frac{1}{m} \frac{d m}{d t}=\frac{d}{d t} \log m
$$

the value of $R$, calculated by formula II, therefore gives correctly the average value of the relative growth rate over the period between the two observations, whatever may be the nature of the changes in relative growth rate over this period. This property of formula II, and the precise definition of relative growth rate at any instant given by III should be borne in mind in considering the confused and contradictory use of the term in the ensuing quotations.

For when we refer to the paper under discussion(4) to ascertain what are the two methods of calculation, the merits of which are to be discussed, we find that the term relative growth rate is now applied to a quantity calculated as a schoolboy is tanght to calculate Simple Interest. The justification of this procedure must be quoted in full ((4), p. 104).

There are various methods of presenting the results, and in the first instance we shall use the relative growth rate curve. The principle of the proposed method of expressing rate of growth is analogous to that of the method by which the rate of most reactions, both chemical and physiological, are expressed, namely amount of change per unit of material per unit of time. Since the amount of material in the growing plant is constantly changing, and since the relative rate of growth is not constant, as the following analysis will show, to achieve mathematical accuracy, the increase should be measured over an infinitely short period. This procedure is manifestly impossible, and as we have no exact knowledge of the way in which the relative rate of growth varies, over a given period we have adopted the following purely conventional method of defining relative rate of growth. The relative rate of growth of a plant during any given week of its life-cycle, is the amount of dry matter which $100 \mathrm{gms}$. of dry matter taken at the beginning of the week adds during the week. A week has been ehosen since this is the usual interval between determinations of dry weight in most experiments on growth in plants. It must be noticed that the method does not pretend to mathematical accuracy, being merely an approximate average for the week, but with such results as are at present available nothing more accurate can be obtained."

The most striking feature of this paragraph is the contrast between the precision of the first definition of relative growth rate contrasted with the inconsequent arbitrariness of the method proposed for its calculation. Nothing could be more clear than to define relative growth rate as "amount of change" (i.e. increase) "per unit of material per unit of time"; the statement is equivalent to that of expression III. But why, if so precise a definition can be given of the quantity we wish 
to measure, should we be reduced to measuring it by a "purely conventional method" which "does not pretend to mathematical accuracy?" The reasons given for this falling away are quite inadequate. It is true that "to achiove mathematical accuracy," in determining the rate of growth at any instant, "the increase should be measured over an infinitely short period"; it should also be measured with infinite accuracy, and on an infinite sample of plants. But the discussion being devoted to the quantitative analysis of such data as Kreusler supplies, it is not clear what would be gained if we could determine the relative growth rate with mathematical accuracy at any one instant. The environmental data quoted by Briggs, Kidd and West are the Mean Terrperatures for the week, and in some cases the total hours of sunshine. Both of these may be regarded as mean values of quantities which vary in an unknown manner during the week. The corresponding quantity which we require for comparison with them is the mean value of the relative growth rate, which is given with mathematical accuracy by formula II. The difficulty of obtaining the relative rate of increase with high accuracy at any instant thus hardly justifies the complaint that "nothing more accurate can be obtained" than a "purely conventional method," which in fact introduces errors up to 100 per cent. or more!

For the Simple Interest formula employed

$$
100 \times \frac{m_{2}-m_{1}}{m_{1}\left(t_{2}-t_{1}\right)}
$$

is extremely inaccurate in relation to the data to which it is applied. The principal cause of this inaccuracy is that the dry mass of the active plant is assumed to have throughout the week that value that is assigned to it at the beginning of the week. It often happens that during the week the mass has more than doubled; in such cases the relative rate of increase is much exaggerated by reckoning the increase on the initial mass. This error has nothing to do with the assumption of linear increase, for if the rate of increase during the week be assumed to be linear, the mean value of the mass for this period will be

$$
\frac{1}{2}\left(m_{1}+m_{2}\right)
$$

and the rate of increase reckoned on this mean mass, gives a much better approximation. The formula obtained in this way

$$
100 \times \frac{2\left(m_{2}-m_{1}\right)}{\left(m_{1}+m_{2}\right)\left(t_{2}-t_{1}\right)}
$$

is compared with that of Briggs, Kidd and West in the following example ((4), p. 107). 


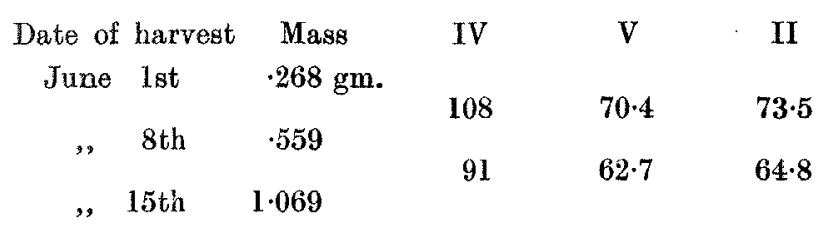

Column IV shows the values given by Briggs, Kidd and West, column $\mathrm{V}$ the values obtained from the approximate formula $\mathrm{V}$, which would be appropriate for the assumption of linear increase ${ }^{1}$, column II gives the true values of formula II; for comparison all are written as percentages. It will be observed that in the first instance IV is 34.5 in excess of the true value, while $V$ is $3 \cdot 1$ in defect, in the second instance IV is $26 \cdot 2$ in excess, and $V$ is $2 \cdot 1$ in defect. The greater part of the error in IV is due to reckoning the increase upon the initial mass.

The errors produced in this way are very irregular; for positive values the rates of increase are exaggerated, for negative values they are made unduly small; so that if in one week the mass is diminished, and in the second it is increased to its former value, the rate of decrease calculated by formula IV will be smaller than the rate of increase which has exactly counterbalanced it. For very small values the formula is approximately accurate. Applied to such data as those presented its irregularities render it most unsuitable for statistical treatment.

But another irregularity is introduced by Briggs, Kidd and West. In the words of a footnote ((4), p. 105), "Where results are not given for a week, we have calculated the increase per $100 \mathrm{gms}$. for the period, and divided the result by the number of weeks in the period." Such a procedure would indeed be accurate, if the true measure of relative rate of increase had been chosen. Its effect upon the grow th rate as estimated by formula IV may best be shown by recalculating the growth rate in the above example, ignoring the value of June 8 th. Then for the whole fortnight:

\begin{tabular}{cccccc} 
& \multicolumn{4}{c}{ Relative rate of increase per wee } \\
\cline { 3 - 5 } & & IV & V & II \\
June Ist & $.268 \mathrm{gm}$. & & & \\
, 15th & 1.069 & $149 \cdot 4$ & 59.9 & $69 \cdot 2$
\end{tabular}

The average weekly rate of increase for the fortnight as calculated by the principles of Simple Interest is thus much greater than that of either of the weeks composing it. The value given by formula $\mathrm{V}$, though

1 In the case of linear increase, the relative growth rate necessarily diminishes, having its highest value at the beginning and its lowest at the end of the period. Column IV then shows the value at the beginning, $V$ the value at the centre of the period, and II, in this as in other cases shows the mean value over the whole period. 
not so ridiculously discordant, shows that the error of this formula increases rapidly as the time interval is increased, that of formula III alone gives a concordant result, being the mean of the values of the two component weeks.

This example brings out a point in the utility of II which is worth noting. It illustrates the fact that growth rates calculated by 11 may be expressed at once in any unit of time, irrespective of the intervals employed experimentally. In general it is most suitable to use the day as the unit, and for this purpose the values in column II need only be divided by 7 . For the values of IV a dilemma arises; according to the method of correction quoted above, they also should be divided by 7 , although this would lead to values inconsistent with any possible daily increases in weight; the only self-consistent method would be to reckon the interest payable daily on the principles of compound interest, and this if performed by the use of logarithms amounts to calculating the value of column II from that of IV, dividing by 7 , and then finding the corresponding value of column IV. This process should be in itself of considerable educative value in the study of these different methods of measurement.

These being the facts, it remains to enquire how it was that with Blackman's work before them, and knowing that the efficiency index had been successfully applied (2), the authors of (4) chose to employ so inaccurate a method of calculation. The point is dealt with in the following passage ((4), p. 106).

"It might be suggested that allowance could easily be made for the continuous increase in dry weight during the week by assuming that this takes place at a uniform rate, and consequently that by means of the following logarithmic formula the rate could be determined:

$$
\log W-\log W_{0}=\mathbf{r},
$$

where $W$ is the dry weight at the end of the week, and $W_{0}$ the dry weight at the beginning of the week.

In curve A, Fig. 1 , this allowance has been made. In curve B, the ordinates are relative growth rates calculated by our method, that is, without making allowance for the continuous increase during the week. The curves show similar variations of growth rate from week to week. The more complicated method, however, does not achieve accuracy, as it rests on the assumption that the rate remains constant during the week, an assumption manifestly incorrect since the rate varies from week to week. Both methods are purely conventional and only approximate to accuracy, and nothing definite is to be gained by adopting the more complicated procedure."

At first sight one might judge from this paragraph that the two methods of calculation had been judged to be practically equivalent by an inspection of the diagram referred to, but a glance at that diagram is sufficient to show that this cannot be so, for while the disagreement is 
inconsiderable for the periods of slow increase or decrease, at the time of most rapid growth the formula ultimately adopted is approximately double the true value; whence it must be apparent that any quantitative study of the growth rates is materially influenced by the choice of the measure of growth rate. Nor can the difficulty of calculation explain the choice, for with the aid of a table of natural logarithms the correct value is more quickly calculated than any of its approximations; indeed it is surprising that the experience of calculating one series did not bring conviction on this point. One can only explain the choice by the mistaken impression, which is emphasised above, that the use of the logarithmic formula involved the assumption that the relative rate of increase is independent of the time; an assumption which as the authors state, is manifestly incorrect.

This conclusion is confirmed by an apparently disconnected discussion, with which the paper opens, of the general mathematical formulae which have been surgested to represent the growth history of annual plants: among them there is ascribed to Blackman the view that this growth history can be represented by an exponential curve, that is to say, by a constant relative growth rate. Whether this view of the growth history of annual plants has ever been advocated, I am unable to say, but there can be no doubt that in advocating the use of accurate methods for measuring relative growth rate, Blackman(1) is amply justified.

\section{SUMmaRY.}

The methods of calculation formulated by Briggs, Kidd and West for the analysis of plant growth are inaccurate, (i) in introducing a large exaggeration when the plant is increasing in mass, (ii) in applying to periods of varying length a method of calculation which thereby becomes self-inconsistent.

The correct measure for the mean value of the relative growth rate over any period, long or short, is that advocated by Blackman under the name of the "efficiency index."

\section{REFERENCES.}

(1) Blackman, H. V. (1919). The compound interest law and plant growth. Anna/s of Botany, xxxir. 353.

(2) Brenomer, W. E. (1920). On the relations between growth and the environ. mental conditions of temperature and bright sunshine. Annals of Applied Biology, vI. 211.

(3) West, C., Briaas, G. E., and Krod, F. (1920). Methods and Significant Relations in the quantitative analysis of plant growth. New Phytologist, xrx. 200.

(4) Bargas, G. E., Krod, F., and WEsT, C. (1920). A quantitative analysis of plant growth. Part 1. Annals of Applied Biology, vr. 103. 